\title{
A Case of Cardiac Amyloidosis Diagnosed Using Combination of Echocardiography and Electrocardiogram
}

\section{Ceng Wang}

Zhejiang Provincial People's Hospital https://orcid.org/0000-0001-6780-8934

Jing Wang ( $\square$ wangjing@hmc.edu.cn)

Zhejiang Provincial People's Hospital

\section{Yi Zheng}

Zhejiang Provincial People's Hospital

\section{Tingting Sun}

Zhejiang Provincial People's Hospital

\section{Case report}

Keywords: Cardiac amyloidosis, electrocardiogram, echocardiography

Posted Date: September 15th, 2021

DOl: https://doi.org/10.21203/rs.3.rs-842122/v1

License: (c) (i) This work is licensed under a Creative Commons Attribution 4.0 International License. Read Full License 


\section{Abstract}

Cardiac amyloidosis (CA) is an invasive cardiomyopathy with high mortality. The gold standard of diagnosis is usually cardiac biopsy, but its clinical application is reduced because it is an expensive and invasive procedure. The combination of electrocardiogram and echocardiography, which is associated with typical characteristics, is used to diagnose CA. However, in recent years, this procedure has been less widely used domestically and internationally. The aim of this study was to elaborate on the diagnostic merits of the two methods.

\section{Background}

Amyloidosis is an invasive disease caused by the deposition of amyloid protein in the extracellular matrix. When the cardiomyocytes are involved, it is called cardiac amyloidosis (CA), which often results in diffuse thickening of the ventricular wall, manifested as restrictive cardiomyopathy. CA is a restrictive infiltrative cardiomyopathy associated with high mortality. The gold standard for diagnosis of CA is the presence of amyloid in Congo red-staining of myocardial biopsy, but the invasive biopsy is risky and expensive, making it unacceptable to most patients. A case of CA diagnosed with echocardiography and electrocardiogram in our hospital is reported here.

\section{Case Presentation}

\section{Brief history of present illness}

The patient was a 63-year-old male. He had chest tightness, shortness of breath after physical exertion in the previous month, Later, the chest tightness gradually worsened, and bilateral lower limb edema appeared in the previous two weeks, accompanied by nocturnal paroxysmal dyspnea, weakness.

\section{In-hospital check}

ECG showed sinus rhythm, ST segment and T wave changes, left deviation of electric axis, low voltage (absolute value of lead voltage of most limbs $\leq 0.5 \mathrm{mv}$ ). Echocardiography showed double atrial enlargement, diffuse thickening of the left ventricular wall and right ventricular wall; enhanced echo, the thickest part was about $24 \mathrm{~mm}$. The LVEF was about $57 \%$, the impairment of left ventricular diastolic function (peak $\mathrm{E}$ was greater than twice peak $\mathrm{A}$ ) and massive pericardial cavity effusion (The thickest part was about $27 \mathrm{~mm}$ ). Pericardial puncture was then performed to decompress the effusion. No cancer cells were found. The results of coronary angiography during the period showed no significant stenosis in any of the three vessels.

During the period of admission, symptomatic supportive treatment was given, and the volume of effusion was significantly reduced. The patient was discharged on his own request. However, in about twenty days, he was re-admitted to hospital. The electrocardiogram and Echocardiography after admission was similar to the last time. Based on these presentations, the doctor in-charge combined with other systemic 
symptoms, queried myocardial amyloidosis and suggested that the patient should have a myocardial biopsy for proper diagnosis. However, the patient refused this procedure due to economic conditions. On the next day, the patient had sudden respiratory and cardiac arrest in the ward, and despite active attempts at resuscitation, he died.

\section{Discussion}

Literature reports indicate that primary systemic amyloidosis is a rare disease with poor prognosis ${ }^{[1]}$. It is usually caused by abnormal proliferation of plasma cells, and most often it involves the heart, kidney and skin soft tissue. Restrictive cardiomyopathy and refractory heart failure are the main manifestations when the heart is involved.

In this study, It is worth noting that there was no serious obstruction in each branch of coronary angiography, The electrocardiogram limb lead QRS voltage did not match the thickness of the chamber wall, and the chamber wall was obviously hypertrophic ${ }^{[2]}$, but the limb lead was low voltage, which is a typical "quality/electricity" contradiction. This feature is an important clue in diagnosing CA. Researchers have established that when the ratio of RI voltage to left ventricular posterior wall thickness is $<0.4$, the sensitivity and specificity of primary CA are 91 and $100 \%$, respectively. Moreover, when this ratio is $<0.36$, the sensitivity and specificity of primary CA are 97 and $89 \%$, respectively.

The appearance of myocardial amyloidosis in echocardiography is morphologically characterized by diffuse hypertrophy of the myocardium and increased echogenicity ${ }^{[3]}$, scattered patchy or scintillating granule, including decreased diastolic function of the heart, decreased ejection fraction, systemic edema caused by heart failure, multiple serous cavity effusion (pericardium, thorax and abdominal cavity); pulmonary hypertension, and widened inferior vena cava. The typical manifestations on electrocardiogram are limb lead low voltage, T wave, ST segment change, and chest lead false infarct waveform.

A clear diagnosis of CA depends largely on endomyocardial biopsy. Congo red staining indicates positive, but this procedure is expensive and highly risky. Besides, some basic hospitals cannot carry it out. Thus, by the time the cause is identified, it is usually late and treatment options are limited. The classification of $\mathrm{CA}$ is related to the clinical symptoms. The survival of CA patients is low, their mortality is high, and the prognosis is poor, especially in primary CA. Once the symptoms of heart failure, usually restrictive cardiomyopathy, begin to appear, conventional heart failure medications may not be effective. The monitoring of daily fluid intake and diuretic dose is key to managing CA-induced heart failure. Cardiac involvement has become a serious risk factor affecting the prognosis of the disease. In recent years, a number of clinical studies in China and abroad have confirmed that good clinical benefits for the diagnosis and differential diagnosis of CA can result from linking the parameters of echocardiography and electrocardiogram (two well-established and widely used cardiovascular techniques), with comprehensive analysis of "progressive refractory heart failure + " quality/electricity "contradiction + 
ventricular wall hypertrophy and flare sign" based on medical history ${ }^{[4]}$. Early detection, early diagnosis and early treatment are important for improving the prognosis.

\section{Declarations}

Ethics approval and consent to participate $\otimes$ For this type of study formal consent is not required.

Consent for publication $₫$ Consent for publication was obtained for every individual person's data included in the study.

Competing interests $₫ T$ The authors declare that they have no conflict of interest.

Funding $₫$ This research was awarded Zhejiang Traditional Chinese Medicine Administrationthe(2018ZQ005) and Zhejiang Provincial Health Science and Technology Project Plan (2021 KY062). 区The fundings conceived and designed the manuscript $\$.

Authors' contributions and Acknowledgements $₫ \mathrm{CW}$ and JW conceived and designed the study. $\mathrm{YZ}$ and TTS looked up relevant information. CW wrote the paper. JW, YZ and T-TS reviewed and edited the manuscript. All authors read and approved the manuscript.

\section{References}

1. Clemmensen Tor Skibsted,Eiskjær Hans,Mikkelsen Fabian et al. Left Ventricular Pressure-StrainDerived Myocardial Work at Rest and during Exercise in Patients with Cardiac Amyloidosis.[J] .J Am Soc Echocardiogr, 2020, 33: 573-582.

2. Xu Tiantian,Hu Hongjie,A case report on transient global ventricular wall thickening secondary to acute myocarditis: Focus on the unique role of cardiac MRI.[J] .Medicine (Baltimore), 2020, 99: e19223.

3. Ito Takahide,Suwa Michihiro,Echocardiographic tissue imaging evaluation of myocardial characteristics and function in cardiomyopathies.[J] .Heart Fail Rev, 2021 Jul;26(4):813-828.

4. M. Grogan, A. Dispenzieri, M.A. Gertz, Light-chain cardiac amyloidosis: strategies to promote early diagnosis and cardiac response, Heart 103 (2017) 1065-1072. 